\title{
Ultraluminous X-ray source XMMUJ132218.3-164247 is in fact a type I Quasar ${ }^{\star}$ (Research Note)
}

\author{
M. Dadina ${ }^{1}$, N. Masetti ${ }^{1}$, M. Cappi ${ }^{1}$, G. Malaguti ${ }^{1}$, G. Miniutti ${ }^{2}$, G. Ponti ${ }^{3,4}$, P. Gandhi $^{5,6}$, and B. De Marco ${ }^{2,4}$ \\ ${ }^{1}$ INAF/IASF Bologna, via P. Gobetti 101, 40129 Bologna, Italy \\ e-mail: mauro.dadina@iasfbo.inaf.it \\ 2 Centro de Astrobiología (CSIC-INTA), Dep. de Astrofisica, PO Box 78, 28691 Villanueva de la Cañada, Madrid, Spain \\ 3 Faculty of Physical and Applied Science, University of Southampton, Southampton SO17 1BJ, UK \\ ${ }^{4}$ Max Planck Institut für extraterrestrische Physik, Giessenbachstrasse 1, 85748 Garching, Germany \\ 5 Institute of Space and Astronautical Science (ISAS), Japan Aerospace Exploration Agency, 3-1-1 Yoshinodai, Chuo-ku, \\ Sagamihara, 252-5210 Kanagawa, Japan \\ ${ }^{6}$ Department of Physics, Durham University, South Road, Durham DH1 3LE, UK
}

Received 7 December 2012 / Accepted 14 September 2013

\begin{abstract}
Context. The true nature of ultraluminous X-ray sources (ULX) is still currently vigorously debated. One of the most interesting possibilities is that these sources are intermediate-mass $\left(M \sim 10^{3}-10^{5} M_{\odot}\right)$ black holes. The most luminous ULX $\left(L_{2-10 \mathrm{keV}} \sim\right.$ $10^{41} \mathrm{erg} \mathrm{s}^{-1}$ ) are the most suitable candidates for being genuine intermediate-mass black holes (IMBH).

Aims. XMMUJ132218.3-164247 was proposed to be the most luminous ULX known so far. For this reason a firm assessment of its intrinsic nature is needed and pursued in this work.

Methods. We precisely defined the position of XMMUJ132218.3-164247 using a short Chandra pointing of the field. Then, we obtained high quality optical spectra of the source with the FORS2 instrument on the VLT to define its nature and distance. We then used unpublished data obtained with XMM-Newton to investigate its nature and emission properties in more detail.

Results. Features in its optical spectrum place XMMUJ132218.3-164247 at a redshift of $z \sim 1$, implying that it is a background QSO instead of an ULX. We clearly detected some emission lines typical of a QSO, including OIII lines and a broad Mg II line that indicates that the source is a type I AGN. The X-ray spectrum of the source is well modeled by a simple power-law with absorption slightly in excess to the Galactic value. No emission feature at the energy of the $\mathrm{FeK} \alpha$ is present in the data. Finally, the source has been detected at a X-ray flux level higher (by $\sim 40 \%$ ) than previously measured and reported in its discovery work.

Conclusions. XMMUJ132218.3-164247 is not a ULX but a type I QSO. It shows a standard X-ray spectrum and exhibits a variability pattern that is typical of QSOs. A very rough estimate of its black-hole mass yields a value of $M_{\mathrm{BH}} \sim 2 \times 10^{7} M_{\odot}$.
\end{abstract}

Key words. galaxies: distances and redshifts - galaxies: nuclei - galaxies: active

\section{Introduction}

Ultraluminous X-ray sources (ULX) are extragalactic X-ray point sources located off-nucleus from their host galaxies. Their bolometric luminosities exceed $\sim 10^{39} \mathrm{erg} \mathrm{s}^{-1}$ (Fabbiano 1989, 2006; Roberts et al. 2004). These extreme luminosities, assuming isotropic emission at (or below) the Eddington limit, would imply accreting black holes $(\mathrm{BH})$ with masses of $\sim 10^{3}-10^{4}$ solar masses. The existence of such intermediate-mass black holes (IMBH) is in dispute. ESO 243-49 HLX-1 (Farrell et al. 2009; Wiersema et al. 2010; Farrell et al. 2012) is perhaps the only ULX case for which a consensus for IMBH has been reached to date. None of the other IMBH candidates, however, is widely accepted as definitive (see for instance the discussion about the mass of NGC 5408 X-1, Casella et al. 2008; Middleton et al. 2011; Dheeraj \& Strohmayer 2012).

^ Based on observations collected at the European Organization for Astronomical Research in the Southern Hemisphere, Chile (program 079.D-0299(A)) and on observations obtained with $X M M-N e w t o n$, an ESA science mission with instruments and contributions directly funded by ESA Member States and NASA.
One important aspect is therefore to exclude that ULX could be background AGN, by using optical spectra (see for example the case of NGC 4168-ULX1, Masetti et al. 2003, and XMMU J124825.9+083020, Foschini et al. 2002a,b).

In this context we present the analysis of optical and X-ray data of the ULX XMMUJ132218.3-164247. The target was observed in X-rays for the first time during an XMM-Newton pointing of the type II Seyfert galaxy IRAS 13197-1627 (also known as MCG-03-34-64). XMMUJ132218.3-164247 was the secondstrongest X-ray source in the field of view with an X-ray flux of $F_{0.5-10 \mathrm{keV}} \sim 10^{-13} \mathrm{erg} \mathrm{s}^{-1} \mathrm{~cm}^{-2}$. The source was detected within the $D_{25}$ ellipse of nearby galaxy MCG-03-34-63 (see upper left panel of Fig. 1). If it is located at the distance of this galaxy, the implied luminosity of XMMUJ132218.3-164247 is $L_{0.5-10 \mathrm{keV}} \sim 10^{41} \mathrm{erg} \mathrm{s}^{-1}$. Considering also the variability observed in its X-ray light curve, the source could have been detected at up to $L_{0.5-10 \mathrm{keV}} \sim 1.5 \times 10^{41} \mathrm{erg} \mathrm{s}^{-1}$ (Miniutti et al. 2006). The intrinsic nature of the source was tentatively investigated by Miniutti et al. (2006) using the data available at the time. In particular, the extreme $F_{\mathrm{x}} / F_{\mathrm{o}} \sim 90$ ratio suggested that the source was most probably not a background AGN 

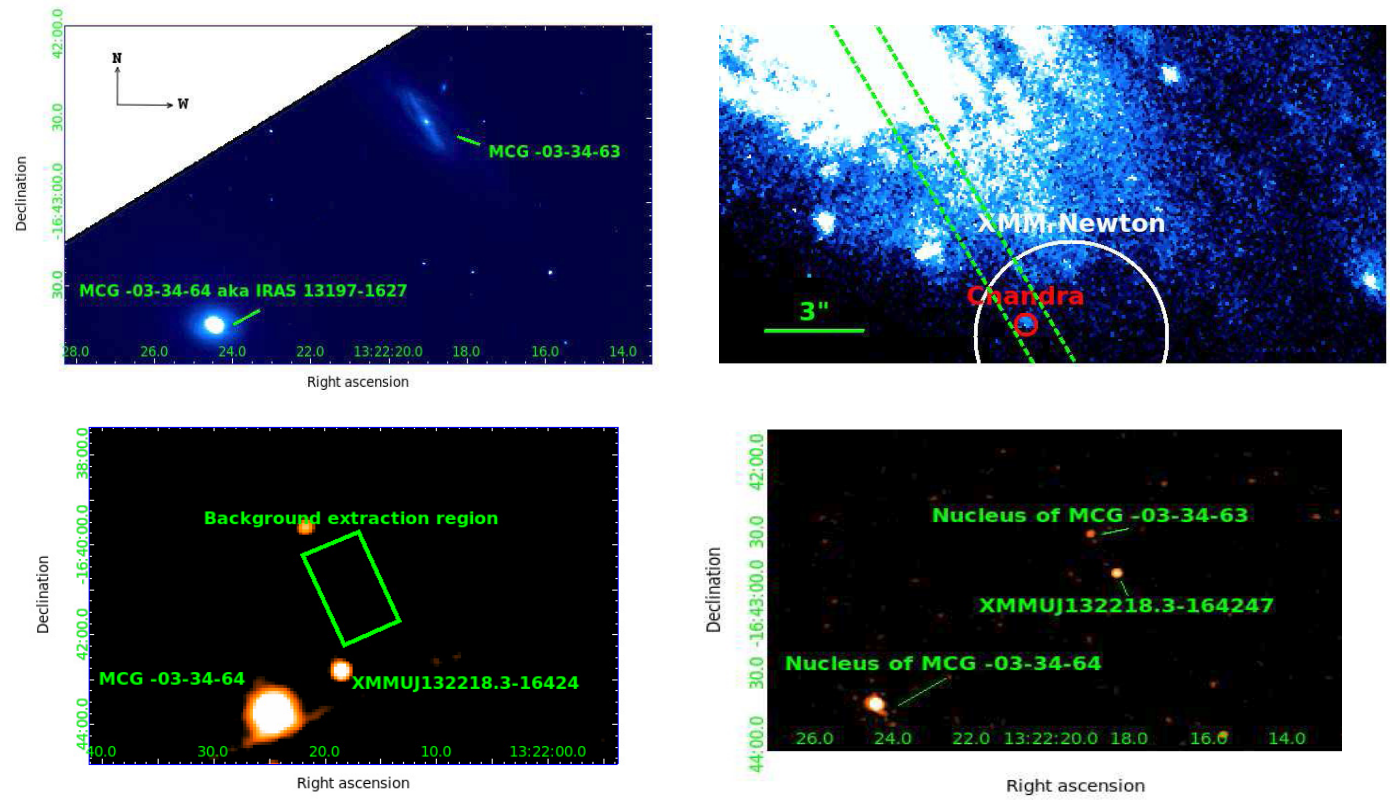

Fig. 1. Upper left panel: $R$-band acquisition image taken with FORS2 CCD1. The two brighter galaxies are IRAS 13197-1627 (MCG-3-34-64) on the left/east and MCG-3-34-63 on the top/north. The images are all oriented in the same way. Upper right panel: HST image obtained with the W606 filter of MCG-3-34-64. The position of XMMUJ132218.3-16424 is indicated by the Chandra error circle in red (the white circle was the XMM-Newton error circle) while the dashed green lines indicate the position of the slit during the FORS spectral observation. As stated in the text, the ULX candidate, once the astrometry is calibrated using USNO-B1 catalogs, falls within the Chandra error circle. The W606 magnitude of the target has been recorded to be $m_{F 606}=24.5$. Lower panels: XMMUJ132218.3-16424 field as seen by XMM-Newton (left panel) and Chandra (right panel). In the XMM-Newton field the background extraction region for the pn is also displayed.

$\left(\log \left(F_{\mathrm{X}} / F_{\mathrm{O}}\right)=\log F_{\mathrm{X}}+0.4 R+5.61\right.$, where $F_{\mathrm{X}}$ is the $2-10 \mathrm{keV}$ flux and $R$ is the magnitude in the $R$ band, see Miniutti et al. 2006). Here we report on the redshift measurement of the source and with this precisely identify it as a QSO.

\section{Observation log and data reduction}

MCG-03-34-63 was observed with Chandra ACIS for $7 \mathrm{ks}$ on July 31, 2006. We ran the source detection tool wavedetect on the Chandra data and found that XMMUJ132218.3164247 is at RA 13:22:18.343 and Dec $-16: 42: 46.91$ (J2000). However, we also checked the accuracy of the Chandra astrometry for this particular observation. The only other clearly detected source in the vicinity of XMMUJ132218.3-164247 is the relatively bright AGN IRAS 13197-1627, about $1.8^{\prime}$ away from XMMUJ132218.3-164247. We have cross-correlated the Chandra position for the AGN with the USNO-B1.0 optical catalogue to find that the Chandra RA and Dec are both displaced by $\sim 0.3^{\prime \prime}$. We therefore corrected the output of the wavedetect tool and found that the accurate X-ray position of XMMUJ132218.3-164247 is RA 13:22:18.365 and Dec -16:42:47.20 (J2000). The cross-correlation with the AGN IRAS 13197-1627 allowed to reduce the nominal Chandra error-circle from $0.6^{\prime \prime}$ in radius to a much more stringent $0.3^{\prime \prime}$. The Chandra position falls well within the XMM-Newton errorcircle as reported in Miniutti et al. (2006). As reported there, the field was pointed by Hubble Space Telescope (HST) in 1994 for $500 \mathrm{~s}$. After correcting the HST astrometry by using five common sources with the USNO-B1.0 catalog, we found that the source that was indicated as the counterpart (Miniutti et al. 2006) has coordinates RA 13:22:18.341 and Dec-16:42:47.00, well within the Chandra error circle (see right panel of Fig. 1).

The VLT observed XMMUJ132218.3-164247 with the FORS2 instrument for a total of $6 \mathrm{~h} 36 \mathrm{~m}$. We took fifteen spectra
Table 1. Observation log.

\begin{tabular}{lcc}
\hline \hline Instrument & Date & Exp. \\
\hline Chandra ACIS & July 31, 2006 & $7 \mathrm{ks}$ \\
FORS2 & April 11, 2007 & $6 \times 480 \mathrm{~s}$ \\
& & $9 \times 1000 \mathrm{~s}$ \\
& & total 12 ks \\
& & $41 \mathrm{ks}$ \\
EPIC pn & January 24, 2008 & $46 \mathrm{ks}$ \\
EPIC MOS 1 & & $41 \mathrm{ks}$ \\
EPIC MOS 2 & & \\
\hline
\end{tabular}

of the source on April 11, 2007 starting at around 03:00 UT (see Table 1).

The observation was performed in service mode and had to last three hours, but the pointing was longer because of the variable seeing. We summed all the data to obtain the highest possible signal-to-noise ratio. Because of the faintness of the source, we used the lowest resolution grism (150I) without order-sorting filters, affording a spectral coverage in the useable range of $\sim 4000-10500 \AA$. The slit width was $1^{\prime \prime}$ for all the observations, and this set-up secured a resulting spectral resolution of $R=\lambda / \Delta \lambda=250$ at $\sim 5000 \AA$. The spectra were extracted within the IRAF ${ }^{1}$ environment. After correcting for flat-field and bias, the spectra were background subtracted and optimally extracted (Horne 1986). Wavelength and flux calibration of the spectra were obtained by using $\mathrm{He}-\mathrm{Ne}-\mathrm{Ar}$ and $\mathrm{Hg}-\mathrm{Cd}$ lamps and by observing the spectrophotometric standard star LTT6248 (Hamuy et al. 1994). The resulting spectrum is shown in Fig. 2.

The XMM-Newton data were taken on January 24, 2008 for $\sim 41 \mathrm{ks}$. The EPIC instruments on-board XMM-Newton were operated in full window mode with the medium filter applied.

http://iraf.noao.edu/ 


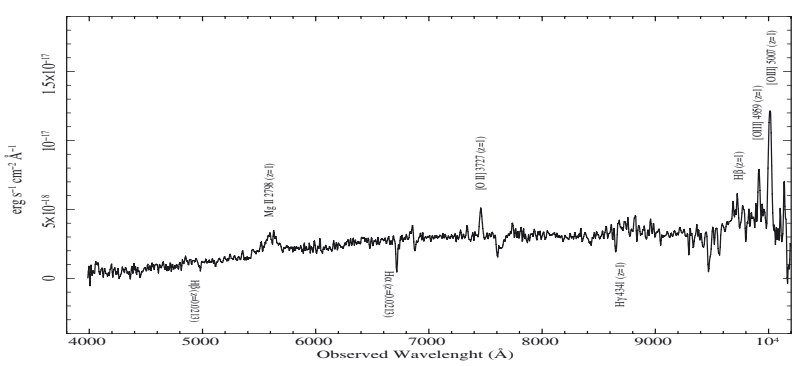

Fig. 2. Average optical spectrum of XMMUJ132218.3-164247. The main spectral features are labeled. The absorption features at $\sim 6900$ and $\sim 7600 \AA$ are telluric.

The raw data were cleaned using $\mathrm{SAS}^{2}$ version 12.0 and inspected for high background level periods induced by soft protons. The overall exposure was $\sim 41 \mathrm{ks}$ (see Table 1) for EPIC pn and 41 and $46 \mathrm{ks}$ for EPIC MOS cameras 1 and 2. The X-ray spectrum of XMMUJ132218.3-164247 was extracted from a circular region centered on the source and with a radius of $22^{\prime \prime}$ and $20^{\prime \prime}$ for EPIC pn and MOS respectively. The pn/MOS background has been extracted from large rectangular/circular regions in the same chips of the target (see lower left image of Fig. 1 for the EPIC/pn case). The data were then cleaned for high background periods that were present at the end of the observation.

\section{Optical classification}

The average spectrum taken at VLT with the FORS2 instrument is shown in Fig. 2. It displays a number of emission lines that can be readily identified with typical optical emission from an AGN. Among these we can detect the [OIII] doublet $(\lambda=4959$ and $5007 \AA$ restframe). Moreover, we also identify the [OII] line at $3727 \AA$ restframe and some hints of a broad components of $\mathrm{H}_{\beta}$ and $\mathrm{H}_{\gamma}$. Unambiguously identified as a broad emission line is the $\mathrm{Mg}$ II emission feature at $2798 \AA$ restframe. After fitting with a single Gaussian, the width of this line is $\sigma \sim 95 \AA$, corresponding to a $F W H M \sim 2700 \mathrm{~km} \mathrm{~s}^{-1}$ at the redshift of the source. All these emission lines are found at a wavelength that is twice of its restframe value, which indicates a redshift $z \sim 1$. To exclude all, we also analyzed the spectrum of the nucleus of MCG-03-34-63 (see upper left panel of Fig. 1), finding that no similar features are present and confirming that these emission lines are not caused by high-order contamination. Averaging the value obtained from the [OIII], [OII] and $\mathrm{Mg}$ II lines, we finally obtained a redshift of $z=1.006 \pm 0.003$. This shows that XMMUJ132218.3-164247 is in fact a background type I QSO.

Following McLure \& Jarvis (2002), we use the width of the MgII line and the UV flux at $3000 \AA$ (restframe) to infer the mass of the SMBH of the QSO. When we correct the observed flux at $6000 \AA$ for the absorption caused by our own Galaxy, we obtain that the $\mathrm{BH}$ has $M \sim 2 \times 10^{7} M_{\odot}$ (we used $H_{0}=73 \mathrm{~km} \mathrm{~s}^{-1} \mathrm{Mpc}^{-1}, \Omega_{\mathrm{m}}=0.27$ and $\left.\Omega_{\Lambda}=0.73\right)$. It is worth noting here that this must be considered only as rough estimate since it does not account for any possible intervening additional absorption component that may alter the UV flux estimation. That there is such a component is suggested by the curvature of the spectrum below $\sim 6000 \AA$ and may explain the observed $F_{\mathrm{O}} / F_{\mathrm{X}}$. The quality of the data, moreover, prevents a detailed modeling of the broad $\mathrm{MgII}$ emission line, and the error

\footnotetext{
2 http://xmm.esa.int/sas/
}

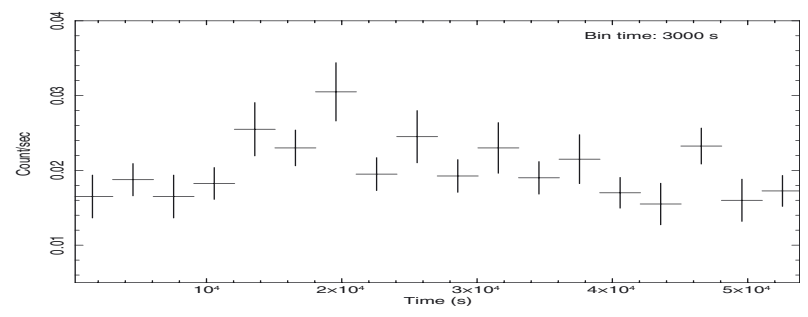

Fig. 3. $0.3-10 \mathrm{keV}$ light curve of XMMUJ132218.3-164247 as observed by XMM-Newton on January 24, 2008.

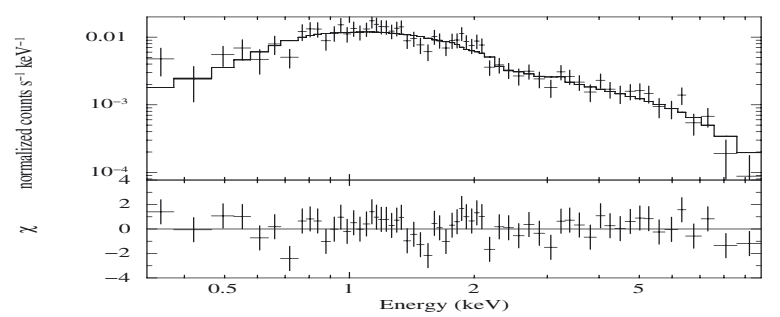

Fig. 4. 0.3-10 keV spectrum of XMMUJ132218.3-164247 as observed by XMM-Newton on January 24, 2008.

associated to its width is on the order of $\sim 50 \%$. Furthermore, the $\mathrm{H}_{\alpha}$ and $\mathrm{H}_{\beta}$ absorption lines measured at the redshift of the foreground MCG-03-34-63 (see Fig. 1) clearly indicate that part of the measured UV flux is probably contaminated by the stellar component of the galaxy itself. Finally, it has been demonstrated that the MgII estimator may easily misestimates the mass of the central SMBH by a factor of $\sim 3$ (McGill et al. 2008).

We also tried to refine the spectral classification taking advantage of the detection of the $\mathrm{H}_{\beta}$ emissione line. The broad component of this line has a complex structure (see Fig. 2), and given the data quality, only a crude estimate of its flux can be obtained. After fitting with a single Gaussian, we obtained a ratio between $\mathrm{H}_{\beta} /[\mathrm{OIII}] \sim 2-3$. This result suggests that the source may be a type 1.2 object according to the classification of Winkler (1992).

\section{New X-ray spectrum}

The high-energy emission of the source displays some variability (see Fig. 3). Its steadiness is rejected at more than the $99 \%$ confidence level. The variability of the source was highlighted by Miniutti et al. (2006).

The X-ray spectrum of the source is well modeled by an absorbed power-law (see Fig. 2 and Table 2). An emission FeK $\alpha$ line is not required by the data $\left(\Delta \chi^{2} \leq 1\right)$. The low energy cutoff detected below $\sim 1 \mathrm{keV}$ (observer frame, Fig. 4), however, indicates that there is an absorption column in addition to the Galactic one $\left(N_{\mathrm{H}}=4.98 \times 10^{20} \mathrm{~cm}^{-2}\right.$, Dickey \& Lockman 1990).

The measured $N_{\mathrm{H}}$ of the extra absorber depends on the redshift at which we assume it to be located (first and second row of Table 2, respectively). MCG-03-34-63 intercepts the line of sight, which may add an additional absorption component. We therefore tested the cases in which the absorber is in MCG-03-34-63 or in XMMUJ132218.3-164247. The quality of the X-ray data prevents us from testing the case in which both objects contribute to the absorption. The fit slightly improved when the absorber is located at the redshift of XMMUJ132218.3-164247 (see Table 2 and Fig. 5), but this improvement is not statistically significant. 
Table 2. Simple power-law models for the X-ray spectrum of XMMUJ132218.3-164247: best-fit parameters assuming different positions of the absorbing column density.

\begin{tabular}{lcccccc}
\hline \hline $\begin{array}{l}N_{\mathrm{H}} \\
10^{22} \mathrm{~cm}^{-2}\end{array}$ & $z\left(N_{\mathrm{H}}\right)$ & $\Gamma$ & $\begin{array}{c}E_{\mathrm{FeK} \alpha} \\
\mathrm{keV}\end{array}$ & $\begin{array}{c}E W \\
\mathrm{eV}\end{array}$ & $\begin{array}{c}F_{2-10 \mathrm{keV}} \\
10^{-13} \mathrm{erg} \mathrm{cm}^{-2} \mathrm{~s}^{-1}\end{array}$ & $\chi^{2} /$ d.o.f. \\
\hline $0.76_{-0.20}^{+0.23}$ & $\equiv 1$ & $1.52_{-0.11}^{+0.12}$ & $\equiv 6.4$ & $\leq 124$ & 1.07 & $87.5 / 95$ \\
$0.19_{-0.05}^{+0.05}$ & $\equiv 0.0213$ & $1.57_{-0.12}^{+0.12}$ & $\equiv 6.4$ & $\leq 124$ & 1.07 & $88.5 / 95$ \\
\hline
\end{tabular}

Notes. $N_{\mathrm{H}}$ : measured absorbing column. $z\left(N_{\mathrm{H}}\right)$, redshift at which the absorbing matter is assumed to be. $\Gamma$ : X-ray photon index. $E_{\mathrm{FeK} \alpha}:$ rest-frame energy of the emission line. EW: emission line equivalent width. $F_{2-10 \mathrm{kev}}$ : observed flux in the $2-10 \mathrm{keV}$ band. $\chi^{2} /$ d.o.f.: $\chi^{2} /$ degrees of freedom.

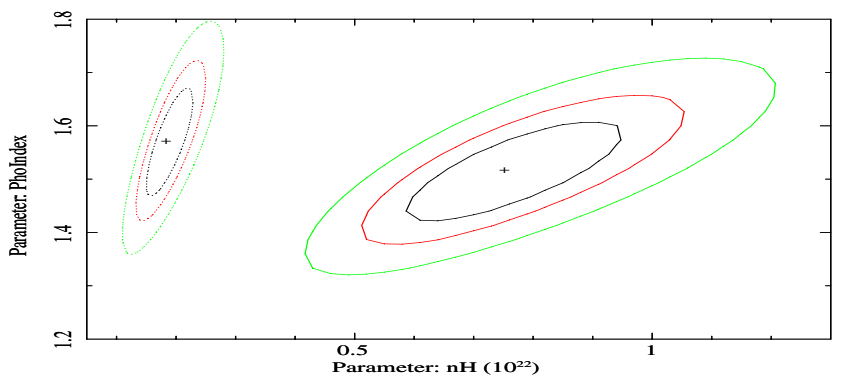

Fig. 5. $\Gamma$ vs. $N_{\mathrm{H}}$ contours assuming the absorber to be at $z=0.0213$ (dotted contours) and at $z=1$ (solid lines). Note that both values are not consistent with, and exceed at more then $99 \%$ confidence level, the Galactic column $\left(N_{\mathrm{H}}=4.98 \times 10^{20} \mathrm{~cm}^{-2}\right.$, Dickey \& Lockman 1992).

Finally, the X-ray spectrum of the source is flat $(\Gamma \sim 1.6)$ comparing with nearby AGNs $(\Gamma \sim 1.8-2$; Dadina 2008$)$ but, when the errors are considered, it is consistent with what have been found for high- $z$ quasars $(\Gamma=1.6 \pm 0.4$ Cappi et al. 1997 ; $\Gamma=1.86_{-0.37}^{+0.41}$ Vignali et al. 2003). This value, however, is not consistent with the one previously measured by Miniutti et al. (2006) at a time when the source was weaker and displayed a steeper spectrum $\left(F_{0.5-7 \mathrm{keV}} \sim 8 \times 10^{-14} \mathrm{erg} \mathrm{s}^{-1} \mathrm{~cm}^{-2}\right.$, while in $2008 F_{0.5-7 \mathrm{keV}} \sim 1.2 \times 10^{-13} \mathrm{erg} \mathrm{s}^{-1} \mathrm{~cm}^{-2}$ and $\Gamma \sim 1.95$ in 2006).

\section{Discussion and conclusions}

We presented the analysis of optical and X-ray observations of the ULX candidate XMMUJ132218.3-164247.

The optical spectrum taken with the FORS2 instrument on the VLT clearly demonstrates that the source is a background QSO at $z \sim 1$. The new X-ray data presented here confirm the variability of the X-ray emission previously reported in Miniutti et al. (2006). Here the source was observed to be in a brighter state and with a flatter spectrum. The source is undoubtely a type I object, even though it displays a certain amount of absorption in X-rays. We speculate that the $[\mathrm{OIII}] / \mathrm{H}_{\beta}$ ratio may indicate that the source is a type 1.2 object. If so, we can expect a certain amount of nuclear absorption to be detected in X-rays (Bassani et al. 1999; Panessa et al. 2006).

We took advantage of the new optical data to obtain a rough estimate of the SMBH mass, which is $M \sim 2 \times 10^{7} M_{\odot}$. As previously stated, this value is only a very rough estimate.

We argue that the absorption explains the $F_{\mathrm{X}} / F_{\mathrm{O}}$ ratio measured using HST data by Miniutti et al. (2006). The observed magnitude in $R$ corresponds to an intrinsic magnitude $m_{R}=20.4$ when we assume that the column measured in X-rays also affects the optical. From this, we found a $A_{v} \sim 4.2$ and, consequently, we obtained that $F_{\mathrm{X}} / F_{\mathrm{O}} \sim 6$, which is not as extreme as previously reported in Miniutti et al. (2006). Again, the assumptions made above are rather simplistic because it has been demonstrated that the absorption measured in X-rays and in the optical and IR are often different (Maiolino et al. 2001).

Acknowledgements. We thank the referee, Matt Malkan, for his comments that improved the manuscript. M.D. warmly thanks Eliana Palazzi and Elisabetta Maiorano for their helpful comments and their help in reducing and analyzing the optical data. This project has been funded by the INAF PRIN 2011. G.P. acknowledge support via an EU Marie Curie Intra-European fellowship under contract no. FP-PEOPLE-2012-IEF-331095.

\section{References}

Bassani, L., Dadina, M., Maiolino, R., et al. 1999, ApJS, 121, 473 Cappi, M., Matsuoka, M., Comastri, A., et al. 1997, ApJ, 478, 492 Casella, P., Ponti, G., Patruno, A., et al. 2008, MNRAS, 387, 1707 Dadina, M. 2008, A\&A, 485, 417

Dheeraj, P. R., \& Strohmayer, T. E. 2012, ApJ, 753, 139

Dickey, J. M., \& Lockman, F. J. 1990, ARA\&A, 28, 215

Elvis, M., Wilkes, B. J., McDowell, J. C., et al. 1994, ApJS, 95, 1

Fabbiano, G. 1989, ARA\&A, 27, 87

Fabbiano, G. 2006, ARA\&A, 44, 323

Farrell, S. A., Webb, N. A., Barret, D., Godet, O., \& Rodrigues, J. M. 2009, Nature, 460, 73

Farrell, S. A., Servillat, M., Pforr, J., et al. 2012, ApJ, 747, L13

Feng, H., \& Soria, R. 2011, New Astron. Rev., 55, 166

Feng, H., Rao, F., \& Kaaret, P. 2010, ApJ, 710, L137

Foschini, L., Di Cocco, G., Ho, L. C., et al. 2002a, A\&A, 392, 817

Foschini, L., Ho, L. C., Masetti, N., et al. 2002b, A\&A, 396, 787

Frank, J., \& Rees, M. J. 1976, MNRAS, 176, 633

Fryer, C. L., \& Kalogera, V. 2001, ApJ, 554, 548

Hamuy, M., Suntzeff, N. B., Heathcote, S. R., et al. 1994, PASP, 106, 566

Maiolino, R., Marconi, A., Salvati, M., et al. 2001, A\&A, 365, 28

Masetti, N., Foschini, L., Ho, L. C., et al. 2003, A\&A, 406, L27

McGill, K. L., Woo, J.-H., Treu, T., \& Malkan, M. A. 2008, ApJ, 673, 703

McLure, R. J., \& Jarvis, M. J. 2002, MNRAS, 337, 109

Middleton, M. J., Roberts, T. P., Done, C., \& Jackson, F. E. 2011, MNRAS, 411, 644

Miniutti, G., Ponti, G., Dadina, M., et al. 2006, MNRAS, 373, L1

Panessa, F., Bassani, L., Cappi, M., et al. 2006, A\&A, 455, 173

Peterson, B. M., Bentz, M. C., Desroches, L.-B., et al. 2005, ApJ, 632, 799

Portegies Zwart, S. F., Makino, J., McMillan, S. L. W., \& Hut, P. 1999, A\&A, 348, 117

Predehl, P., \& Schmitt, J. H. M. M. 1995, A\&A, 293, 889

Rao, F., Feng, H., \& Kaaret, P. 2010, ApJ, 722, 620

Roberts, T. P., Warwick, R. S., Ward, M. J., \& Goad, M. R. 2004, MNRAS, 349, 1193

Strohmayer, T. E., Mushotzky, R. F., Winter, L., et al. 2007, ApJ, 660, 580

Volonteri, M., Haardt, F., \& Madau, P. 2003, ApJ, 582, 559

Vignali, C., Brandt, W. N., Schneider, D. P., et al. 2003, AJ, 125, 2876

Wiersema, K., Farrell, S. A., Webb, N. A., et al. 2010, ApJ, 721, L102

Winkler, H. 1992, MNRAS, 257, 677 